\title{
Correction to: Kin17 facilitates thyroid cancer cell proliferation, migration, and invasion by activating $\mathrm{p} 38$ MAPK signaling pathway
}

\author{
Qun-Guang Jiang ${ }^{1} \cdot$ Cheng-Feng Xiong ${ }^{2} \cdot$ Yun-Xia Lv ${ }^{2}$
}

Published online: 6 January 2021

(c) Springer Science+Business Media, LLC, part of Springer Nature 2020

Correction to: Molecular and Cellular Biochemistry https://doi.org/10.1007/s1 1010-020-03939-9

The affiliation of the second and third author was incorrectly published in the original article. The correct affiliation appears in this correction.

Publisher's Note Springer Nature remains neutral with regard to jurisdictional claims in published maps and institutional affiliations.

The original article can be found online at https://doi.org/10.1007/ s11010-020-03939-9.

Yun-Xia Lv

ndefy12389@ncu.edu.cn

1 Department of Gastrointestinal Surgery, The First Affiliated Hospital of Nanchang University, Nanchang 330006, Jiangxi Province, People's Republic of China

2 Department of Thyroid Surgery, The Second Affiliated Hospital of Nanchang University, No. 1, Minde Road, Nanchang 330006, Jiangxi Province, People's Republic of China 